\title{
Representation of Multiple Durations in Children and Adults
}

\author{
Maria Grazia Carelli and Helen Forman \\ Department of Psychology, Umeå University, 90187 Umeå, Sweden \\ Correspondence should be addressed to Maria Grazia Carelli, grazia.carelli@psy.umu.se
}

Received 16 August 2011; Revised 14 November 2011; Accepted 18 December 2011

Academic Editor: Jeffrey W. Fagen

Copyright ( 2011 M. G. Carelli and H. Forman. This is an open access article distributed under the Creative Commons Attribution License, which permits unrestricted use, distribution, and reproduction in any medium, provided the original work is properly cited.

\begin{abstract}
Keeping track of durations of multiple event attributes with different onset and offset times is a challenging task for both children and adults. In this study, children between 5 and 15 years and young adults observed a puppet show in which three puppets appeared on the scene during overlapping intervals of $30 \mathrm{~s}$ to $90 \mathrm{~s}$. At test, participants completed a conventional time estimation task and a timeline task in which they reconstructed the temporal pattern by drawing a timeline for each puppet. For all age groups, the timeline task produced more accurate duration judgments than the time estimation task. Preschoolers' time estimation was at chance level, but their timeline performance was surprisingly good and age differences were eliminated in some task conditions. These findings suggest that the timeline procedure provides an efficient retrieval support for complex temporal events and that even preschool-aged children are able to represent multiple asynchronous durations, possibly by relying on relational event knowledge in combination with visuospatial retrieval support.
\end{abstract}

\section{Introduction}

Learning how to translate the subjective experience of time's passing into conventional standardized time units is a challenging task for children. Friedman [1] found that comprehensive skills in the use of conventional time units does not emerge until late to midadolescence. Children typically give very vague accounts when asked to give estimates in such units. Especially younger children often "misuse" conventional standardized time units [2-4]. Pouthas [5] argued that most young children $(<7$ years) have not yet learned how to use conventional time units in a reasonably accurate way.

Temporal understanding is even more challenging, because most everyday activities do not present with a neatly ordered row of events, contained and unfolding one at the time. Rather, most activities are temporally complex and involve asynchronous, partially overlapping events $[6,7]$; see also Zalla et al. [8, 9]. Consider, for example, a theatre play in which the actors constitute the elements of the event (along with props). A theatre play reflects different temporal levels, including the real time of the performance, which commences at a specific hour and typically ends a couple of hours later. Furthermore, most events are composed of subelements, with individual temporal characteristics. In a play, the actors may enter and leave the scene simultaneously or separately, and they may appear for different periods of time.

Although most everyday events are temporally complex, past (psychophysically oriented) timing studies have investigated temporal processing in overly simplified stimulus conditions. Typically, participants observe a discrete event for a few seconds, after which they are instructed to make a judgment of its duration. In most cases, the observed effects (i.e.,over- or underestimations) are consistent with the existing theories of timing such as the attentional-gate model of prospective timing [10-12] and the contextual change model of retrospective timing [13], but psychophysical methods of interval timing are not easily applied to more complex goal-directed activities in everyday situations.

A more valid test of children's mastery of time should, thus, include multiple stimulus durations, arranged in a configuration of partly overlapping and nonoverlapping events. This might be a more authentic reflection of how time is actually experienced outside the laboratory. To the best of our knowledge, only two studies have examined cognitive 
timing in the context of multiple duration judgments [14, 15]. Both studies involved adult participants and tested the hypothesis that prospective timing requires attentional resources and that task-irrelevant temporal information impairs prospective duration judgments. Thus, the primary focus of these studies was on the effects of concurrent temporal load on single-item duration judgments, rather than patterns of temporal information.

Specifically, Brown and West's [14] participants monitored the duration of one to four target stimuli with different onset and offset times. At test, they reproduced one of the durations. The main finding of their study was that the magnitude of prospective timing error increased nonlinearly as the number of stimuli increased from one to four targets. Vanneste and Pouthas [15] extended these findings by showing that these effects were accentuated in older adults.

Past studies have not examined developmental differences in more complex timing tasks involving multiple asynchronous stimulus durations, but consistent with most theories of interval timing (see Block et al. [16], for an overview) preschool-aged and younger children should have great difficulties in keeping track of multiple temporal elements with different onset and offset times. One might even argue that preschool-aged children lack necessary cognitive competence for handling complex event information with multiple durations. On the other hand, most real-world events are complex and dynamic (rather than a static picture on a computer screen) and preschool-aged children seem to demonstrate reasonable temporal orientation when observing (and interacting with) everyday events that comprise overlapping temporal trajectories.

Empirical evidence from more applied settings also support this hypothesis. In legal contexts, children are often asked to make temporal judgments about witnessed events (see Powell et al. [17], for a review). To overcome children's difficulties in temporal processing, investigators often use graphical illustrations to help children in their judgment when events occurred. Typically, these "timelines" comprise a line with temporal markers at both ends, and the child is asked to indicate where on the line a specific event occurred.

The timeline is assumed to facilitate children's temporal judgments in criminal investigations, but it should be noted that its reliability in legal contexts has not been examined in past research. However, Friedman and colleagues ([1821], see also Nelson [22]) have emphasized the importance of using a timing method that does not simply rely on conventional time units (which young children clearly do not understand). For example, Friedman and Kemp [20] found that children as young as 4 years of age were able to judge with some accuracy the times of birthdays and holidays from the past several months when tested with a kind of ruler. In this task, the near end of the ruler represented the recent past and the far end the distant past.

In a related study by Friedman [18], children between 4 and 10 years of age judged the distances of events in the future. Children were shown a picture of a road that stretched from near the viewer to the far distance and asked to point on a spatial scale how far events were in the future. Results showed that 5-year olds were quite accurate and were able to distinguish events that would occur in the coming weeks and months from those that would not occur for many months.

These findings suggest that the timeline task might be a useful method for examining temporal information processing in preschool-aged children. However, it should be noted that the primary focus of these studies was on children's ability to recall the location and sequence of a single event duration, rather than complex patterns of asynchronous stimulus durations.

Recently, Carelli [23] reported a study which examined temporal information processing of complex patterns of partially overlapping stimulus events by using two tasks of temporal processing. Specifically, young adults observed a pantomime in which five actors appeared on the scene at different and partially overlapping periods of time. At an unexpected test, they estimated the duration each actor was seen or reconstructed the temporal pattern of the pantomime by drawing a timeline for each actor. Participants made large errors in the time estimation task, but they provided rather accurate responses by using the timeline as a retrieval support. These findings suggest that temporal processing of complex asynchronous events is a challenging cognitive task but that reliance on visuospatial retrieval support, such as the timeline task, may provide reasonable approximations of complex temporal patterns.

Taken together, these findings suggest the timeline task might be a useful method for overcoming preschool-aged children's difficulties in temporal processing. This hypothesis is also consistent with past studies on children's understanding of symbolic models. These studies (e.g., DeLoache et al. [24]) suggest that pictorial representations may help preschoolers to recall events. These schematic representations may also reduce demands on children's limited working memory resources $[25,26]$ in complex temporal tasks. In other words, consistent with the findings of Carelli (in press), the timeline procedure might facilitate children's temporal processing by reducing cognitive demands in reconstructive retrieval processes in which event durations are inferred by integrating episodic temporal information with knowledge of temporal patterns $[21,22]$.

This notion is also consistent with the hypothesis that representations of multiple durations are based on multiple levels of temporal processing and involve a variety of temporal cues. Instead of internal timekeepers, children (and adults) with limited cognitive resources might rely on a variety of temporal heuristics, including relational knowledge $[27,28]$, task-relevant knowledge structures (e.g., scripts and story schemata [22]), and spatial support systems $[29,30]$. In order to reduce computational demands, these kinds of temporal aids and heuristics might be used to reconstruct and constrain the temporal pattern of the observed event (see also Brown [31] and Friedman et al. [19, 32, 33]).

Following this line of reasoning, we examined temporal processing of asynchronous event information in (preschoolaged and older) children and younger adults by contrasting a traditional, psychophysically oriented times estimation task with a more reconstructive cognitive timing task. Specifically, 
participants first observed a "puppet show," in which three puppets entered and left the scene at different times. At test, participants first completed a time estimation task, in which they estimated the appearance time of each puppet. In the second task, referred as the timeline task, they reproduced the temporal pattern of each puppet show by drawing a timeline for each target object.

Following the findings of Carelli [23], our primary hypothesis was that the time estimation task would produce substantial timing errors for all age groups because asynchronous stimulus durations are not easily encoded in terms of absolute durations (as suggested by the attentionalgate model, e.g., Block and Zakay [10, 11]). Furthermore, we expected significant age effects in the time estimation task, with the 5-year olds showing close to a chance level performance and the 10-year olds performing worse than the older children and the young adults. The time estimation task was included here as a reference measure, while our primary goal was to examine whether temporal processing of complex events could be solved by relying on timelinelike retrieval support. We expected the time estimation task and related measures of interval timing would underestimate children's temporal event knowledge but that the timeline task might serve as more efficient retrieval support also in preschool-aged children. Thus, we reasoned that to the extent that preschoolers have some form of representation of multiple temporal patterns, their performance might be above a chance level in the timeline task which relies on relational knowledge.

\section{Method}

Participants. Eighty-five children and adults participated in the study. Twenty-four children were between 5 and 6 years (12 boys and 12 girls) and 18 children between 9 and 10 years ( 9 boys and 9 girls), and 18 adolescents between 14 and 15 years ( 8 boys and 10 girls). Adult participants $(n=25)$ were between 20 and 31 years $(M=24.4$ years, $S D=4.34)$ with 9 males and 16 females. Children were recruited from an elementary public school, and adult participants were Umeå University undergraduates. All participants spoke Swedish as a first language, and teachers' reports indicated that none of the children had any obvious behavioral or educational problems. Parental consent for participation was obtained for all children.

Stimulus Materials. The stimulus events comprised video recordings of three puppet shows. Each stimulus event included three distinct and unique puppets (e.g., a king, a princess, and a wizard, resp., in Show 1) which appeared on the scene $30 \mathrm{~s}$ (referred to as the short duration), $60 \mathrm{~s}$ (medium duration), or $90 \mathrm{~s}$ (long duration). For example, in Show 1, first the King enters the scene. The King is thinking aloud, expressing how content he is with the order of things. Then the Princess appears and opens a conversation with the King. After a disagreement, the King leaves and the Wizard enters. The Wizard tries to talk some sense into the Princess, but, after failing that, he uses his magic to make her go away.
The Wizard contemplates what just happened and then he also leaves the scene. Different sets of puppets appeared in each show, but the background of each scene was identical for all three stimulus events. Furthermore, the temporal pattern of each show was unique with the constraint that stimulus duration was counterbalanced across the order of appearance. Specifically, across the three shows, each duration appeared as the first, second, and third puppet, respectively. The duration of each show was $120 \mathrm{~s} \pm 3 \mathrm{~s}$. Finally, a $30 \mathrm{~s}$ video with three other puppets was presented during a practice trial.

2.1. Procedure. The experimenter informed the participants that they would be shown a puppet show in which three puppets would be playing on the scene and that they should pay attention how long each puppet appeared on the scene. The experimenter also informed that the play would start when green "start" sign appeared and stop when a red "stop" sign appeared on the screen. The experimenter illustrated the verbal instructions by showing a practice video, followed by the test instructions in which participants were shown all three puppets. Specifically, the 5- and 10-year olds, who were tested individually, were shown the actual puppets, whereas the 15-year olds and the adult participants were given the names of the three puppets. Participants first completed a conventional time estimation task, in which they were instructed to estimate "how long each puppet appeared on the scene." (A separate pilot study suggested minimal effects of test order when the estimation task preceded the timeline task, but not vice versa. Also, as shown in Section 3, the correlation between the time estimation data and timeline data was nonsignificant, suggesting marginal order effects (see also [23])). Participants responded by telling (5- and 10 -year olds) or writing down (older participants) a numeric value in seconds or minutes. In the timeline task, participants were instructed to indicate "how long each puppet appeared on the scene" by drawing a line that corresponded the start and stop times of each puppet. Participants completed the task by using a response sheet with a vertical "start line," which indicated the starting point of the puppet show and three horizontal "tracks" for each puppet. Another vertical "stop line" indicated the total length of the event (without providing any numeric values of its duration). The experimenter explained that the length of the "track" represented the total duration of the puppet show and that participants should estimate when and how long each puppet appeared on the scene by drawing a timeline within each track. Participants were free to order the puppets on the tracks, but most participants listed them in the order of appearance (cf. Figure 1). During the practice trial, the experimenter clarified the test instructions by illustrating the task on a separate response sheet. None of the participants appeared to have difficulties in understanding the instructions. After completing the practice trial, participants viewed the first stimulus video and then completed the timing tasks. The experimenter repeated the instructions before each task and then showed the puppets in order of appearance. The same procedure was repeated for the remaining two videos. 
The timelines format

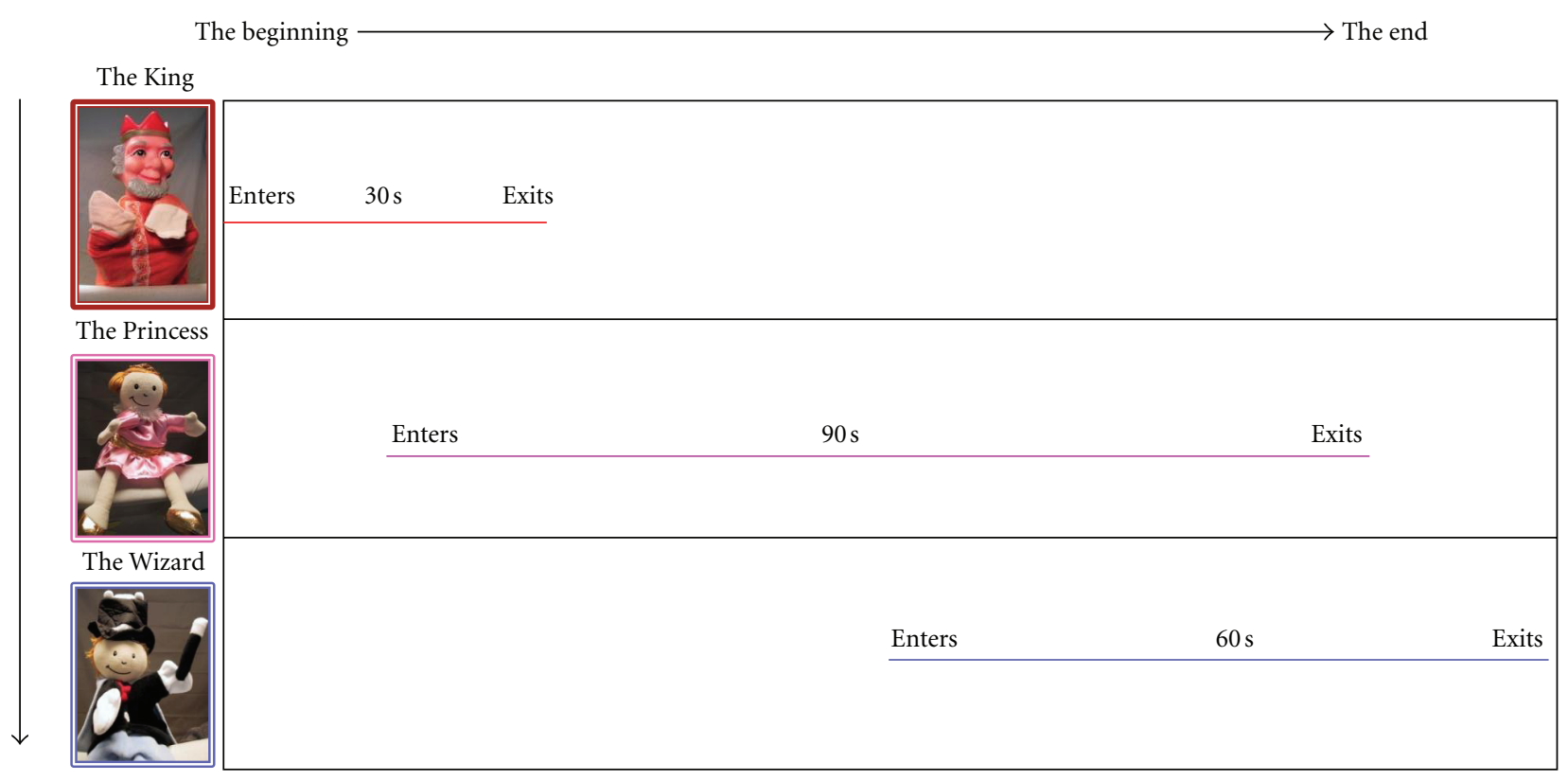

FIGURE 1: The temporal pattern of Show 1.

The maximum response time for each timing task was 5 min, and the whole experiment took about $20 \mathrm{~min}$ to complete.

\section{Results}

The timing data of both tasks were analyzed in terms of timing error by calculating the absolute difference between the observed and actual (objective) durations for each stimulus item. For example, if the actual time was $90 \mathrm{~s}$ and the observed time was $75 \mathrm{~s}$, then the absolute error would have been 15 s (see, e.g., Barkley et al. [34] and also Carelli et al. $[25,35])$. The timeline was obtained by first transforming each response to time units and then calculating timing errors as indicated above. In this transformation, the maximum line length of $240 \mathrm{~mm}$ corresponded the total duration of the $120 \mathrm{~s}$ stimulus event (i.e., $1 \mathrm{~s}=2 \mathrm{~mm}$ ).

Furthermore, the timeline data were based on two measures, referred to as the length and position errors, respectively. The length error of the timeline task is identical to that of time estimation task in that the difference between the observed and actual durations is calculated by comparing the length of the lines. However, this measure does not reflect errors relative to the actual start and stop times (i.e., where the timeline was placed). For example, response A between $5 \mathrm{~s}$ and $100 \mathrm{~s}$ and response B between $50 \mathrm{~s}$ and $145 \mathrm{~s}$ would produce the same duration error (i.e., $15 \mathrm{~s}$ if the actual start and stop times were $20 \mathrm{~s}$ to $100 \mathrm{~s}$, resp.). The position error is more stringent than the duration error in that the start and stop errors are calculated separately. The absolute errors for A would be $20 \mathrm{~s}$, that is, abs(5-20) + abs(100-100), and for B $75 \mathrm{~s}$, that is, abs(50-20) + abs(145-100). Finally, we also examined timeline performance by using a relative error score in which the observed stimulus duration (i.e., line

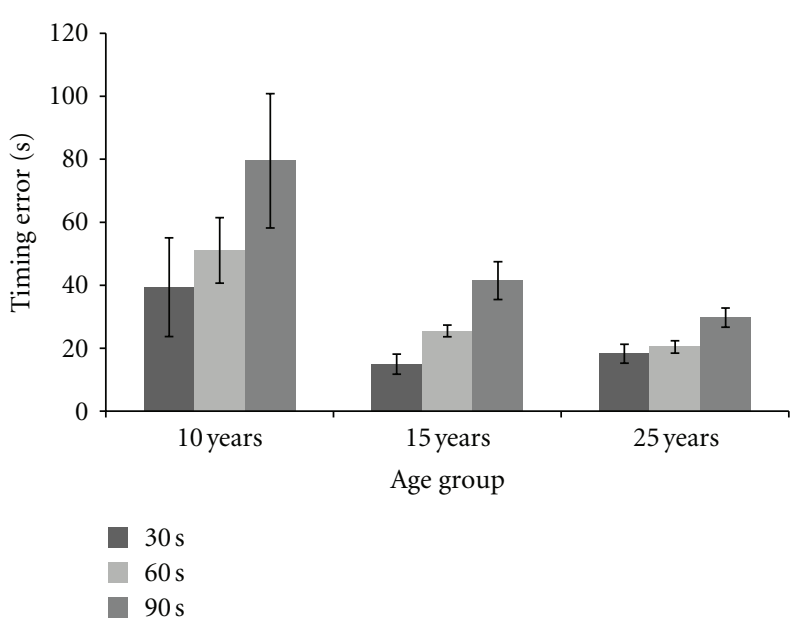

FIGURE 2: Duration error in the time estimation task as function of age group and stimulus duration. Error bars denote one standard error around the mean.

length) was divided by the actual duration. This measure provides a standard score across the different time intervals, with coefficients above 1.0 reflecting overestimations and coefficients below 1.00 reflecting underestimations.

3.1. Time Estimation Data. Figure 2 shows the time estimation data as a function of age and stimulus duration. Preschoolers' data are not shown in Figure 2, because their performance was at chance level. Specifically, most 5-year olds were unable to respond, and those few children who responded made very large timing errors (absolute errors varying between $120 \mathrm{~s}$ and $1770 \mathrm{~s}, M=418 \mathrm{~s}$ ). As displayed in 


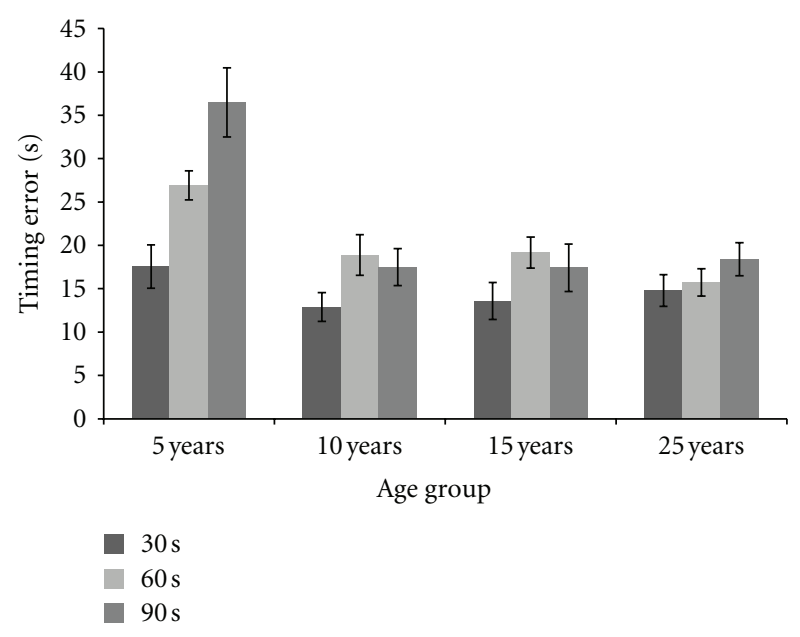

Figure 3: Duration error in the timeline task as a function of age group and stimulus duration. Error bars denote one standard error around the mean.

Figure 2, also 10-year olds made large errors, and the magnitude of their timing error increased as a function of stimulus duration. Adolescents and adults showed similar patterns of performance, with somewhat larger errors for longer durations. These observations were confirmed in a 3 (age) $\times 3$ (duration) mixed analysis of variance (ANOVA), with duration as a within-subjects factor and age group as a betweensubjects factor. Specifically, the ANOVA yielded significant main effects of duration, $F(2,116)=29.32, M S e=373.97$, $\eta_{p}{ }^{2}=0.34, P<.01$, and group, $F(2,58)=4.82$, MSe $=$ 4026.74, $\eta_{p}{ }^{2}=0.14, P<.01$. Also, the interaction between group and duration was significant, $F(4,116)=3.13$, MSe $=$ 373.97, $\eta_{p}{ }^{2}=0.10, P<.02$. Tests of within-subjects contrasts indicated that only the linear trend was significant, $F(2,58)=$ $7.67, M S e=6698.15, \eta_{p}^{2}=0.21, P<.01$. No other effects were observed.

3.2. Timeline Data. Figure 3 shows the mean length error as a function of age group and stimulus duration. Compared to time estimation performance, these data show a clear improvement for all age groups (see also Figure 5). Specifically, the 5-year olds performed at a chance level in the estimation task, but their timeline performance (in terms of length errors) was surprisingly good ( $M=26.39 \mathrm{~s}$, or $5.28 \mathrm{~cm}$ in terms of line length). Similarly, although the 10 -year olds made large errors in the time estimation task, their timeline performance was very similar to that of adolescent and adult participants. Furthermore, compared to the adolescents and adults, the 5-year olds showed excellent performance in the $30 \mathrm{~s}$ condition. As shown in Figure 2, timing error increased as a function of stimulus duration, and this effect was accentuated in preschoolers.

A mixed ANOVA on these data yielded significant main effects of duration, $F(2,162)=13.78, M S e=101.14, \eta_{p}^{2}=$ $0.15, P<.01$, and group, $F(3,81)=11.91, M S e=146.33$, $\eta_{p}{ }^{2}=0.31, P<.01$. Bonferroni post hoc tests showed that the mean of 5 -year olds $(M=26.49)$ was significantly

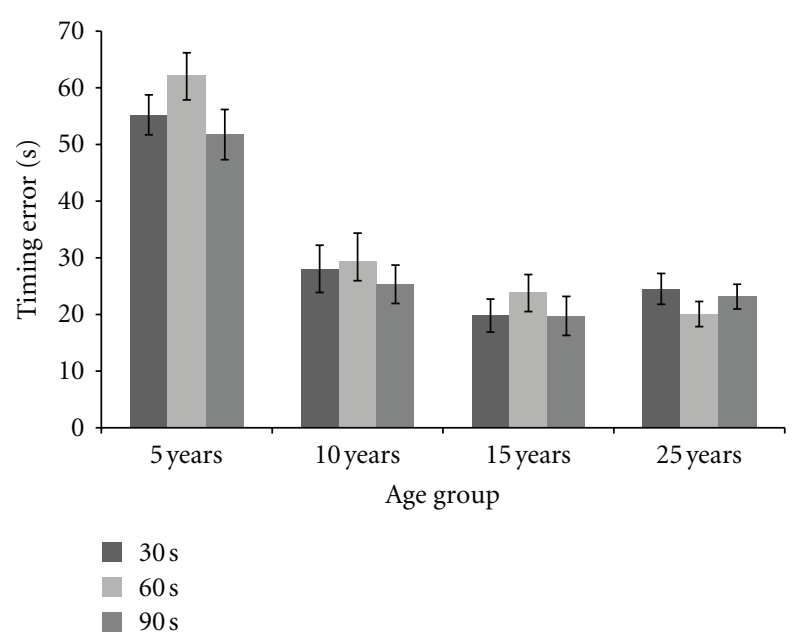

FIgURe 4: Position error in the timeline task as a function of age group and stimulus duration. Error bars denote one standard error around the mean.

larger than those of the older age groups (all $P s<.01$ ), with no differences among them (means varying between 16.30 and 16.64). Furthermore, the interaction between group and duration was significant, $F(6,162)=4.54, M S e=101.14$, $\eta_{p}{ }^{2}=0.14, P<.01$. Subsequent analyses indicated that the group difference was significant in the $90 \mathrm{~s}$ and $60 \mathrm{~s}$ conditions (both $P s<.01$ ), but not in the $30 \mathrm{~s}$ condition $(F<$ $1)$. In other words, the 5 -year olds were equally accurate as the adults and older children when the timeline task involved short durations. No other effects were observed.

We also examined these data in terms of relative errors. The four age groups showed similar patterns of relative errors in that they overestimated the shortest $30 \mathrm{~s}$ duration $(M=$ $1.21)$ and underestimated the $60 \mathrm{~s}(M=0.79)$ and $90 \mathrm{~s}(M=$ $0.85)$ durations, $F(2,162)=55.24, M S e=0.08, \eta_{p}{ }^{2}=0.41$, $P<.01$. Furthermore, the main effect of group was significant, $F(3,81)=4.35, M S e=0.37, \eta_{p}{ }^{2}=0.13, P<.01$, and post hoc tests showed that the mean of 5-year olds $(M=0.84)$ was significantly lower than that of the 25 -year olds $(M=$ $1.01, P<.02)$ and 15 -year olds $(M=1.02, P<.02)$, but not that of the 10 -year olds $(M=0.95)$.

To examine the relation between times estimation and timeline performance, we completed a series of (Pearson's) correlation analyses. These analyses were based on aggregated measures (across durations and stimulus events) and indicated nonsignificant correlations between time estimation and timeline performance (all $r s<.21$ ). We also completed separate analyses for children and adults, but both analyses showed similar patterns of nonsignificant correlations.

Figure 4 shows the position error data as a function of age group and stimulus duration. As expected, these data show somewhat greater errors than the length data, but it should be noted that the 10-year olds show a similar pattern of results as the adolescent and adult participants. Furthermore, the 5-year olds show higher error rate than the other age groups across the three stimulus durations. A mixed ANOVA 


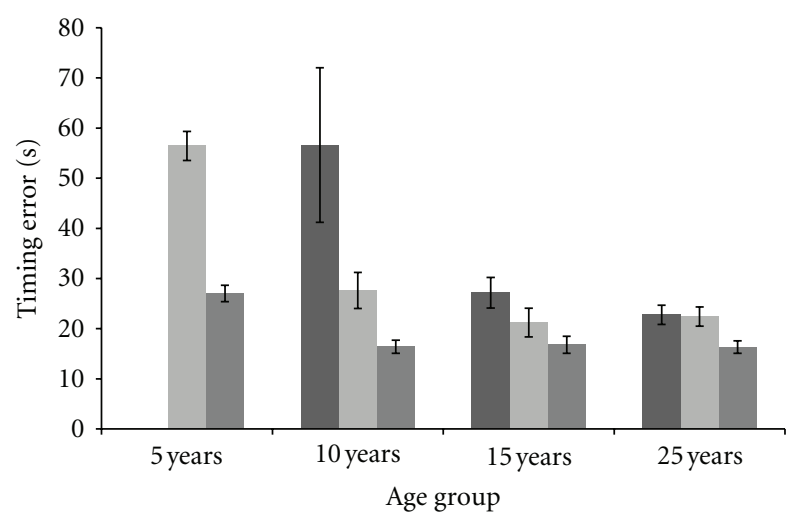

Verbal time estimation

Timelines position

Timelines time estimation

FIGURE 5: Timing performance as a function of age group and type of measure. Error bars denote one standard error around the mean.

confirmed this observation with significant main effects of group, $F(3,81)=35.47, M S e=503.93, \eta_{p}{ }^{2}=0.56, P<.01$. Post hoc tests showed that the mean of the 5 -year olds was significantly larger than those of the older groups $(P<.01)$, with no differences among them.

Finally, Figure 5 illustrates the magnitude of age difference across the three measures. As indicated by the analyses summarized above, this pattern of results clearly shows that the timeline task facilitated retrieval in all age groups. The most striking finding in these data is that the 5 -year olds showed surprisingly good performance in the timeline task and that the 10-year olds performed at the same level as the university undergraduates.

\section{Discussion}

The starting point of this study was the observation that young children typically give very vague and even incorrect accounts when asked to provide duration estimates in conventional units of time. This is a major obstacle in many applied settings, including legal contexts, in which children are often asked to make temporal judgments about witnessed events. As most everyday activities, these events are often structurally and temporally complex. Most episodes involve a complex symphony of asynchronous, partially overlapping events, rather than a neatly ordered row of events, contained and unfolding one at a time.

Temporal information processing of parallel and dynamic activities is not easily handled by the existing models of (prospective or retrospective) duration judgments. For example, the attentional-gate model of Block and Zakay $[10,12]$ makes consistent predictions in task situations which require estimation or reproduction of a relatively short stimulus duration, but is not designed for handling temporal processing of more complex, asynchronous events.

The aim of this study was to investigate preschoolaged and older children's temporal processing of complex, partially overlapping event information. Specifically, we examined developmental differences in temporal processing of asynchronous event attributes by contrasting a traditional, psychophysically oriented time estimation task with a more reconstructive cognitive timing task.

A central finding of this study was that the two timing tasks showed large differences in accuracy, suggesting that temporal processing of multiple event attributes is not easily handled by traditional models and measures of interval timing. Consistent with the studies of Brown and West [14] and Vanneste and Pouthas [15], both children and adults made large timing errors in the estimation task. By contrast, the timeline task increased response accuracy for all age groups, and the age difference in timing error (between 5and 25-year olds!) was virtually eliminated for short stimulus durations. Taken together, these findings suggest that even preschool-aged children are able to reproduce relatively complex patterns of temporal patterns when relying on visuospatial retrieval support.

Although the timeline task appears to facilitate even young children retrieval of temporal event information, the timeline and time estimation tasks have important structural differences in the magnitude of potential errors. Time estimation requires a transformation of duration judgments to verbal estimates (in which children have great difficulties). In the time estimation task these errors can be infinitely large, whereas errors in the timeline task are more constrained by its response format (i.e., the maximum line length). However, it should be noted that two tasks were based on the same encoding phase and study instructions. In both tasks, the target durations were reported simultaneously and could be related to each other and modified during the course of retrieval. Furthermore, the two tasks involved virtually identical scoring procedures with comparable and multiple measures of timing error.

A reasonable interpretation of our findings is that participants reduced working memory demands by relying on relational knowledge of the observed temporal pattern (cf. Halford [27, 28]). Instead of representing the start and stop times of each actor in terms of absolute intervals, a less demanding strategy would be to reconstruct an approximation of these durations, possibly by judging the magnitude of each individual duration relative to each other and whole event. In the timeline task, the participants were instructed to retrieve all the stimuli durations together, and when completing this task they might have drawn on its relational knowledge, which in this task involved three durations. As the notion of relational knowledge suggests [28], when a tertiary relation is to be recreated as a configuration of three different durations, it can easily be broken down into several binary relations, where the individual durations overlap/do not overlap, which makes the procedure less complex and, thus more economic, that is, less cognitively demanding [28].

The second reason for the effectiveness of the timeline format is that it reflects temporal information implicit in the event, which revokes the reliance on standardized time units. According to Nelson [22], most basic concepts of time (e.g., sequence-such as before, after; durationsuch as short time/long time; boundaries of events-such as beginnings and endings) derive and involve relations 
within and among the events in children's everyday life. Most of these concepts and related temporal constructs (e.g., perspective and location) have a direct relation to the everyday experience and memory for events. Our results are consistent with Nelson's theory in that even if preschoolers are not able to translate their temporal knowledge into conventional time units, they still can extract several basic temporal concepts within and between events.

The present findings can also be related to the notion that processing of temporal information is, at least to some extent, mediated by spatial representations. Evidence from psychophysical experiments [29, 36] and psycholinguistic studies [37-39] as well as developmental studies [4, 18, 30, $32,40]$ suggests that people construct spatial representations on line when processing temporal information.

The timeline task, which can be considered as a form of spatial visualization aid, does not require a direct translation of duration experience to conventional units of time, such as seconds and minutes in the time estimation task. Instead, subjective experience of stimulus durations is represented in terms of relative positions, and the resulting pattern of timelines can be used to access more absolute duration estimates. This characteristic of the timeline task might make it particularly suitable for examining temporal information processing in certain populations [20,30].

In conclusion, even preschool-aged children are able to represent multiple temporal patterns, possibly by relying on their relational knowledge in combination with visuospatial retrieval support. We suggest that children in fact do have a rudimentary sense of time, which can be extracted from their knowledge of temporal relations inherent in the flow of events in their everyday life.

\section{Acknowledgments}

This research was supported by a grant from the Swedish Research Council. Portions of this paper were presented at the European Conference of Developmental Psychology (August, 2009, Vilnius, Lithuania).

\section{References}

[1] W. J. Friedman, "The development of children's knowledge of temporal structure," Child development, vol. 57, no. 6, pp. 1386-1400, 1986.

[2] L. Harner, "Children talk about the time and aspects of actions," Child Development, vol. 52, pp. 498-506, 1981.

[3] L. Harner, "Talking about the past and the future," in The Developmental Psychology of Time, W. J. Friedman, Ed., Academic Press, New York, NY, USA, 1982.

[4] R. M. Weist, "Time concepts in language and thought: filling the Piagetian void from two to five years," in Time and Human Cognition, I. Levin and D. Zakay, Eds., pp. 63-118, NorthHolland, Amsterdam, The Netherlands, 1989.

[5] V. Pouthas, "Ontogenesis of temporal learning in the child experimental evidence and perspectives," Psychologica Belgica, vol. 33, pp. 171-183, 1993.

[6] J. M. Zacks and B. Tversky, "Event structure in perception and conception," Psychological Bulletin, vol. 127, no. 1, pp. 3-21, 2001.
[7] J. M. Zacks, N. K. Speer, K. M. Swallow, T. S. Braver, and J. R. Reynolds, "Event perception: a mind-brain perspective," Psychological Bulletin, vol. 133, no. 2, pp. 273-293, 2007.

[8] T. Zalla, P. Pradat-Diehl, and A. Sirigu, "Perception of action boundaries in patients with frontal lobe damage," Neuropsychologia, vol. 41, no. 12, pp. 1619-1627, 2003.

[9] T. Zalla, I. Verlut, N. Franck, D. Puzenat, and A. Sirigu, "Perception of dynamic action in patients with schizophrenia," Psychiatry Research, vol. 128, no. 1, pp. 39-51, 2004.

[10] R. A. Block and D. Zakay, "Models of psychological time revisited," in Time and Mind, H. Helfrich, Ed., pp. 171-195, Hogrefe \& Huber, Kirkland, Wash, USA, 1996.

[11] R. A. Block and D. Zakay, "Prospective and retrospective duration judgments: a meta-analytic review," Psychonomic Bulletin and Review, vol. 4, no. 2, pp. 184-197, 1997.

[12] D. Zakay and R. A. Block, "The role of attention in time estimation processes," Advances in Psychology, vol. 115, pp. 143-164, 1996.

[13] R. A. Block and M. A. Reed, "Remembered duration: evidence for a contextual-change hypothesis," Journal of Experimental Psychology, vol. 4, pp. 656-665, 1978.

[14] S. W. Brown and A. N. West, "Multiple timing and the allocation of attention," Acta Psychologica, vol. 75, no. 2, pp. 103-121, 1990.

[15] S. Vanneste and V. Pouthas, "Timing in aging: the role of attention," Experimental Aging Research, vol. 25, no. 1, pp. 4967, 1999.

[16] R. A. Block, D. Zakay, and P. A. Hancock, "Developmental changes in human duration judgments: a meta-analytic review," Developmental Review, vol. 19, no. 1, pp. 183-211, 1999.

[17] M. B. Powell, K. P. Roberts, and B. Guadagno, "Particularization of child abuse offences: common problems when interviewing child witnesses," Current Issues in Criminal Justice, vol. 19, pp. 64-74, 2007.

[18] W. J. Friedman, "The development of children's knowledge of the times of future events," Child Development, vol. 71, no. 4, pp. 913-932, 2000.

[19] W. J. Friedman, "Memory for the time of past events," Psychological Bulletin, vol. 113, no. 1, pp. 44-66, 1993.

[20] W. J. Friedman and S. Kemp, "The effects of elapsed time and retrieval on young children's judgments of the temporal distances of past events," Cognitive Development, vol. 13, no. 3 , pp. 335-367, 1998.

[21] W. J. Friedman and T. D. Lyon, "Development of temporalreconstructive abilities," Child Development, vol. 76, no. 6, pp. 1202-1216, 2005.

[22] K. Nelson, "The emergence of temporal mind," in Language in Cognitive Development: The Emergence of the Mediated Mind, K. Nelson, Ed., pp. 259-291, Cambridge University Press, Cambridge, UK, 1996.

[23] M. G. Carelli, "Timelines of past events: reconstructive retrieval of temporal patterns," Advances in Cognitive Psychology, vol. 7, pp. 49-54, 2011.

[24] J. S. DeLoache, S. L. Pierroutsakos, and D. H. Uttal, "The origins of pictorial competence," Current Directions in Psychological Science, vol. 12, no. 4, pp. 114-118, 2003.

[25] M. G. Carelli, H. Forman, and T. Mäntylä, "Sense of time and executive functioning in children and adults," Child Neuropsychology, vol. 14, no. 4, pp. 372-386, 2008.

[26] H. Forman, T. Mäntylä, and M. G. Carelli, "Time keeping and working memory development in early adolescence: a 4-year follow-up," Journal of Experimental Child Psychology, vol. 108, no. 1, pp. 170-179, 2011. 
[27] S. G. Halford, "Information-processing models of cognitive development," in Blackwell Handbook of Childhood Cognitive Development, U. Goswami, Ed., pp. 555-575, Blackwell, Malden, Mass, USA, 2002.

[28] G. S. Halford, W. H. Wilson, and S. Phillips, "Relational knowledge: the foundation of higher cognition," Trends in Cognitive Sciences, vol. 14, no. 11, pp. 497-505, 2010.

[29] D. Casasanto and L. Boroditsky, "Time in the mind: using space to think about time," Cognition, vol. 106, no. 2, pp. 579593, 2008.

[30] J. Piaget, The Child's Conception of Time, Ballantine Books, New York, NY, USA, 1927/1969.

[31] S. W. Brown, "Time perception and attention: the effects of prospective versus retrospective paradigms and task demands on perceived duration," Perception \& Psychophysics, vol. 38, no. 2, pp. 115-124, 1985.

[32] W. J. Friedman and S. L. Brudos, "On routes and routines: the early development of spatial and temporal representations," Cognitive Development, vol. 3, no. 2, pp. 167-182, 1988.

[33] W. Friedman, A. G. Gardner, and N. R. E. Zubin, "Childrens comparisons of the recency of two events from the past year," Child Development, vol. 66, pp. 970-983, 1995.

[34] R. A. Barkley, S. Koplowitz, T. Anderson, and M. B. Mcmurray, "Sense of time in children with ADHD: effects of duration, distraction, and stimulant medication," Journal of the International Neuropsychological Society, vol. 3, no. 4, pp. 359-369, 1997.

[35] T. Mäntylä, M. G. Carelli, and H. Forman, "Time monitoring and executive functioning in children and adults," Journal of Experimental Child Psychology, vol. 96, no. 1, pp. 1-19, 2007.

[36] A. Vallesi, M. A. Binns, and T. Shallice, "An effect of spatialtemporal association of response codes: understanding the cognitive representations of time," Cognition, vol. 107, no. 2, pp. 501-527, 2008.

[37] L. Boroditsky, "Metaphoric structuring: understanding time through spatial metaphors," Cognition, vol. 75, no. 1, pp. 128, 2000.

[38] R. Núñez and E. Sweetser, "Looking ahead to the past: convergent evidence from aymara language and gesture in the cross-linguistic comparison of spatial construals of time," Cognitive Science, vol. 30, pp. 401-450, 2006.

[39] B. Tversky, S. Kugelmass, and A. Winter, "Cross-cultural and developmental trends in graphic productions," Cognitive Psychology, vol. 23, no. 4, pp. 515-557, 1991.

[40] I. Lewin, "The development of the concept of time in children: an integrative model," in Time, Action, and Cognition: Toward Bridging the Gap, F. Macar, V. Pouthas, and W. J. Friedman, Eds., pp. 13-32, Kluwer Academic Publishers, Dordrecht, The Netherlands, 1992. 


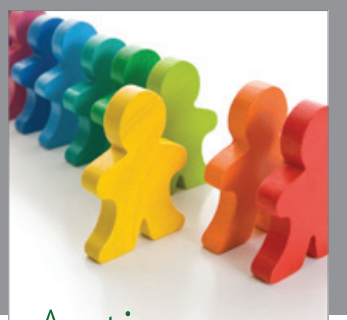

Autism

Research and Treatment
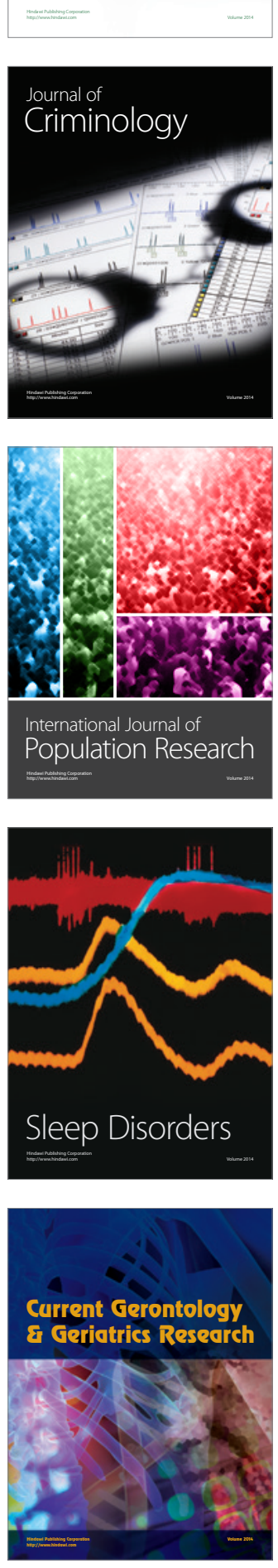
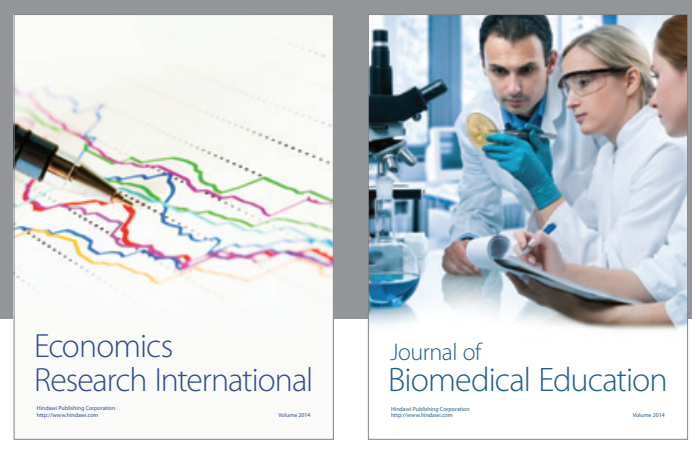

Journal of

Biomedical Education

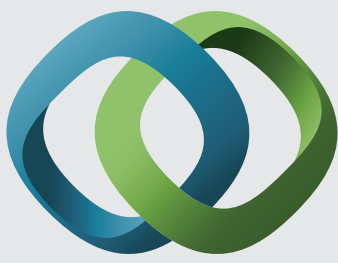

\section{Hindawi}

Submit your manuscripts at

http://www.hindawi.com
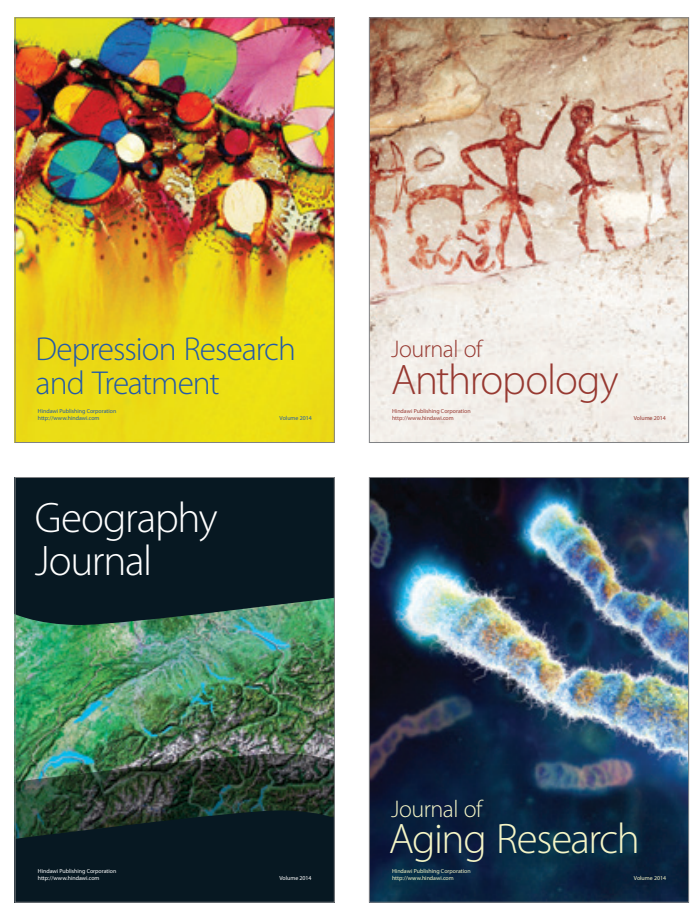

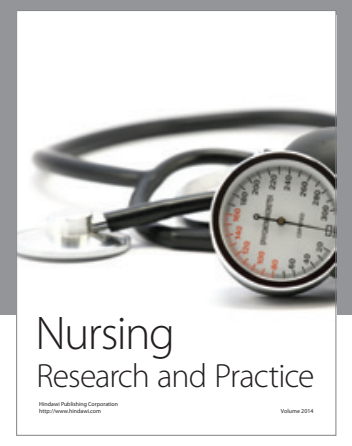

Nursing

Research and Practice

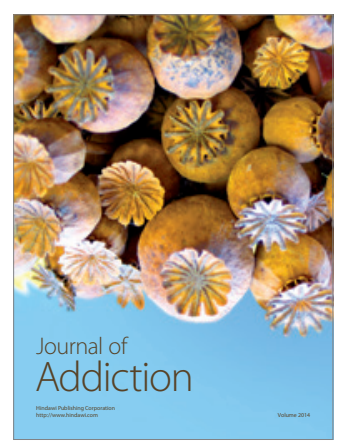

Child Development

Research

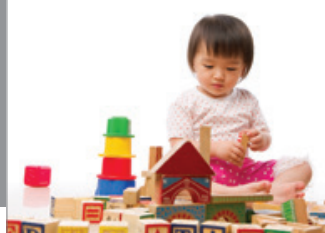

迥
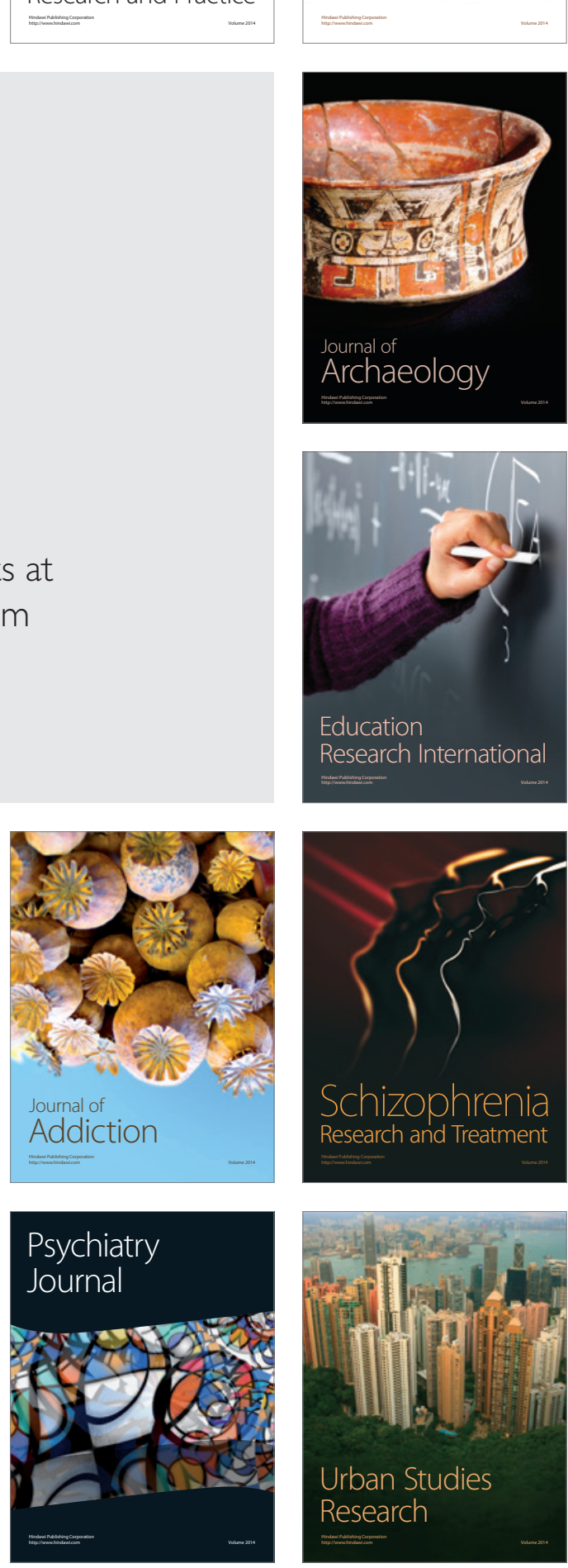